\title{
Design and Fabrication of Slotted Multimode Interference Devices for Chemical and Biological Sensing
}

\author{
M. Mayeh, ${ }^{1,2}$ J. Viegas, ${ }^{1,2,3,4}$ P. Srinivasan, ${ }^{5}$ P. Marques,, 4 J. L. Santos, ${ }^{3,4}$ \\ E. G. Johnson, ${ }^{1,2}$ and F. Farahi ${ }^{1,2}$ \\ ${ }^{1}$ Department of Physics and Optical Science, University of North Carolina at Charlotte, Charlotte, NC 28223, USA \\ ${ }^{2}$ Center for Optoelectronics and Optical Communications, University of North Carolina at Charlotte, NC 28223, USA \\ ${ }^{3}$ INESC Porto, Rua do Campo Alegre 687, 4169-007 Porto, Portugal \\ ${ }^{4}$ Departamento de Física, Faculdade de Ciências da Universidade do Porto, 4169-007 Porto, Portugal \\ ${ }^{5}$ College of Optics and Photonics, University of Central Florida, Orlando, FL 32816, USA
}

Correspondence should be addressed to M. Mayeh, mmayeh@uncc.edu

Received 2 February 2009; Accepted 5 May 2009

Recommended by Christos Riziotis

We present optical sensors based on slotted multimode interference waveguides. The sensor can be tuned to highest sensitivity in the refractive index ranges necessary to detect protein-based molecules or other water-soluble chemical or biological materials. The material of choice is low-loss silicon oxynitride ( $\mathrm{SiON}$ ) which is highly stable to the reactivity with biological agents and processing chemicals. Sensors made with this technology are suited to high volume manufacturing.

Copyright ( $) 2009$ M. Mayeh et al. This is an open access article distributed under the Creative Commons Attribution License, which permits unrestricted use, distribution, and reproduction in any medium, provided the original work is properly cited.

\section{Introduction}

During the last decade there has been a rapidly growing interest in integrated optical sensors because of their many advantages over the nonplanar optical sensors. Many of integrated optical sensors (IOSs) primarily allow for sensitive, real-time, label-free, and on-site measurements of very small changes of biological and chemical parameters of species. There are different methods for IO sensing and the majority of them imply a change of the optical properties of the sensing, area such as its refractive index and thickness, which will be translated into a change in output intensity of optical sensor. In nearly all integrated optical sensors it is the change of effective index of refraction, $N_{\text {eff, }}$, that is utilized, and in some cases measurement methods rely on the phenomenon of optical interference [1]. That is clearly visible in the so-called interferometric sensors in which the output beam of the sensing waveguide interferes with a reference beam. Between the sensors in which they work, based on interference concept, here are a few to mention: Mach-Zehnder interferometers (MZIs) [2-6], Young interferometer [7-9], and Michelson [10], of course resonator-type sensors also rely on interference such as ring resonator [11, 12] and Fabry-Perot resonator [13]. The typical resolution of these classes of sensors is in the order of $10^{-7}-10^{-5}$ refractive index units (RIUs). The most widespread sensing scheme is surface-plasmon resonance (SPR) [14] in which biochemical interaction on the surface of a grating translates into the changes of refractive index and will be monitored by resonance behavior of a guided wave at the surface of a thin metal film. Recently some sensing schemes based on surface-plasmon interference have been proposed $[15,16]$. For these types of sensors typical resolution is in the order of $10^{-6}-10^{-5}$. The grating coupler has been realized in the area of IO sensors as well, using wavelength interrogation or angular interrogation [17-20]. In many cases an improved resolution of the sensor is obtained at the expense of limited operating range.

In this paper a novel type of IO sensor has been introduced. This sensor is based on a slotted multimode interference (MMI) waveguide in which the changes in refractive index are read out as change in output intensity. Due to its compactness, robustness, large operating range, and high sensitivity, the proposed device is a novel contribution to the field of optical sensing. MMI-based optical devices have been widely used for a variety of sensing purposes among them 


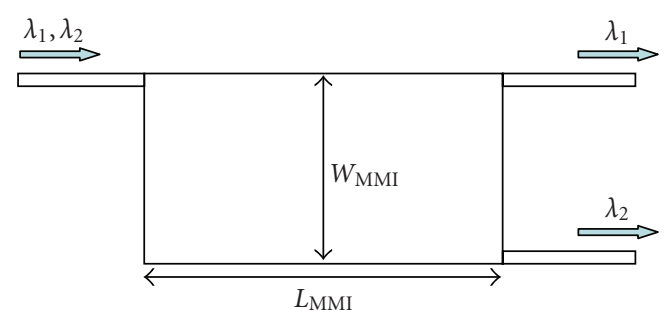

FIGURE 1: Schematic configuration of a, MMI coupler.

temperature [21] and chemical/biological sensors [22, 23] but in the best of our knowledge, slotted MMI devices have never been investigated for sensing applications.

This work describes the design and optimization parameters of the proposed device as well as the detailed description of fabrication process. The performance of the device in terms of sensitivity to bulk refractive index changes of covering medium has been experimentally investigated. The preliminary experimental results demonstrate the applicability of this specific configuration to detection of wide range of chemicals and biological species.

\section{Design}

Multimode interference (MMI) device compromises single mode input and output waveguides and a multimode region. This waveguide region is typically single mode in the transverse direction and multimode in lateral direction. Figure 1 shows a schematic configuration of a simple offcenter-fed MMI [24].

The MMI region supports many lateral modes. The input signal excites these modes, which propagate with different phase velocities along the length of the MMI region and become out of phase. The input field will reproduce itself when the superposition of the modes in the MMI region matches the original modal distribution at the input plane. This condition occurs at planes where the phase differences among the excited modes are integral multiples of $2 \pi$, which allows the excited modes to constructively interfere and reproduce the input's modal distribution. The propagation distance at which this occurs is known as the self-image length and is described in (1):

$$
L_{\pi}=\frac{4 n_{\text {core }} W_{\mathrm{eff}}^{2}}{3 \lambda} .
$$

The self-image length depends upon the wavelength $(\lambda)$ and the polarization of the light, as well as upon parameters of the device such as refractive index $\left(\mathrm{n}_{\text {core }}\right)$ and width $\left(\mathrm{W}_{\text {eff }}\right.$ which is slightly larger than MMI width).

Recently Mackie and lee [25] have introduced a new modification in MMI region which reduces the self- imaging length. The modification involves introducing one or more slots along the entire longitudinal length of the MMI region (Figure 2(b)). The term "slot" refers to a narrow subregion of the MMI region, with a specific width and position that has a different effective refractive index than the rest of the

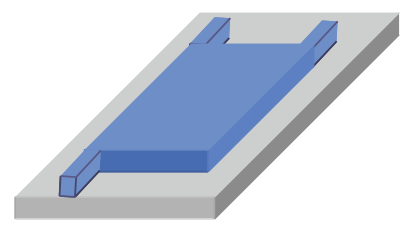

(a)

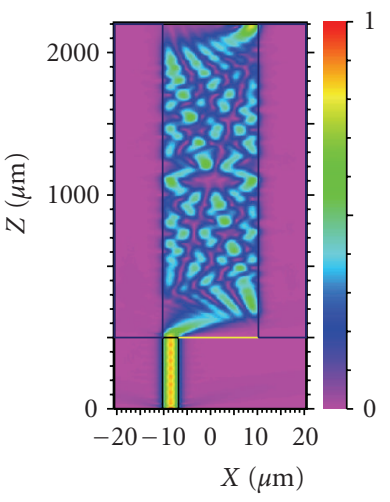

(c)

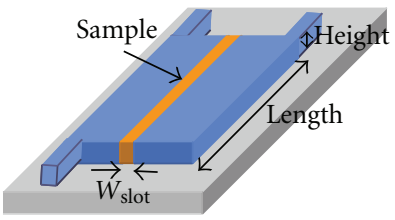

(b)

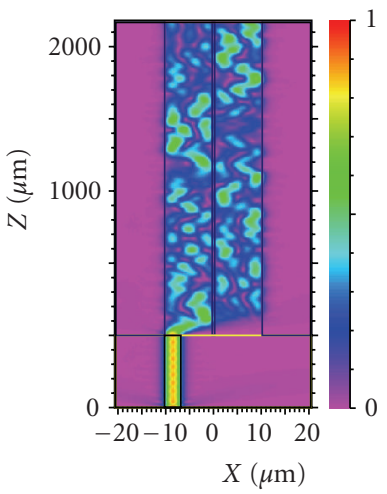

(d)
FIGURE 2: Schematic representation of (a) a simple MMI and (b) a MMI with one slot of width $\left(W_{\text {slot }}\right)$. (c) Top view of field distribution in a MMI device with no slot and (d) one slot.

MMI region. A single centered slot with the right parameters such as refractive index of slot, width and separation of the slot reduces the self-imaging length by a factor of 2 and does so without adversely affecting the performance. Introducing $N$ slots reduces the self-imaging length by a factor of $N+1$. Figures 2(a) and 2(b) represent a simple and one-slot $(N=$ 1) MMI. The field propagation along both devices is depicted in Figures 2(c), and 2(d), assuming a fused quartz wafer as the substrate $\left(n_{\text {clad }}=1.445\right)$ and $\mathrm{SiON}$ as core material $\left(n_{\text {core }}=1.49\right)$. The refractive index of the sample fluid film to be analyzed is 1.45 , and it completely fills the slots. The operating wavelength is $1300 \mathrm{~nm}$, and the width of the slot ( $W_{\text {slot }}$ ) is set to $520 \mathrm{~nm}$. Length of the MMI region (Length) is $2.2 \mathrm{~mm}$, and the height of waveguide is to $2.5 \mu \mathrm{m}$.

As each mode of the MMI region propagates, it acquires a modal phase delay, given by (2):

$$
\phi_{m}=\frac{\pi \cdot p^{2} z}{L_{\pi}},
$$

where $p$ is the mode number, $z$ is the propagation distance, and $L_{\pi}$ is the self-imaging distance for the MMI without a slot. For slotted MMI regions, each mode also acquires an extra phase delay from the slots. This extra phase delay depends upon $z, \delta n$ (the difference in effective refractive index between the slot region and the rest of the MMI region), the slot width $W_{\text {slot, }}$ and the value of the mode across the slot. One can approximate the last parameter as the value of the mode at the center of the slot. In fact, the extra phase delay from a slot will be zero for modes that have nodes (i.e., are zero) at that slot position. Self-imaging 


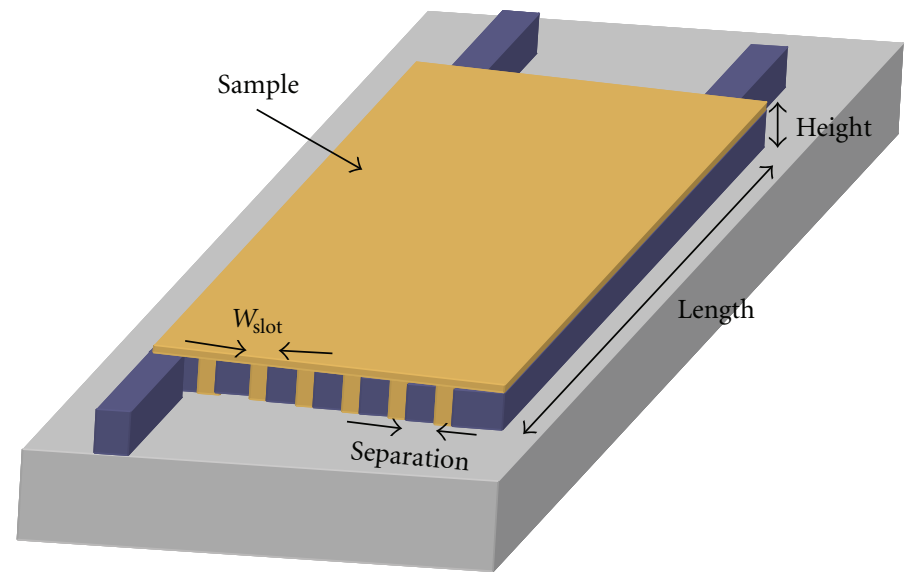

FIGURE 3: Schematic representation of a MMI with six-slots of equal widths $\left(W_{\text {slot }}\right)$ which are separated by equal distances.

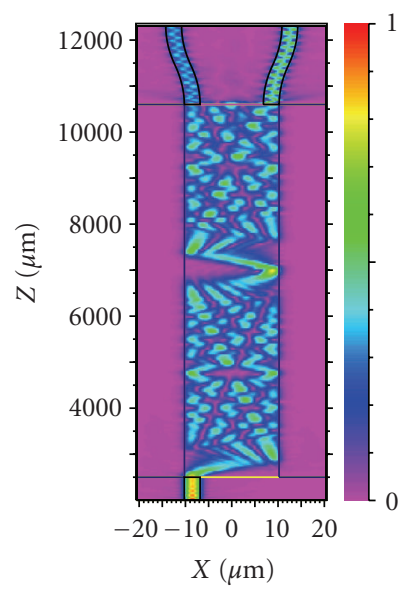

(a)

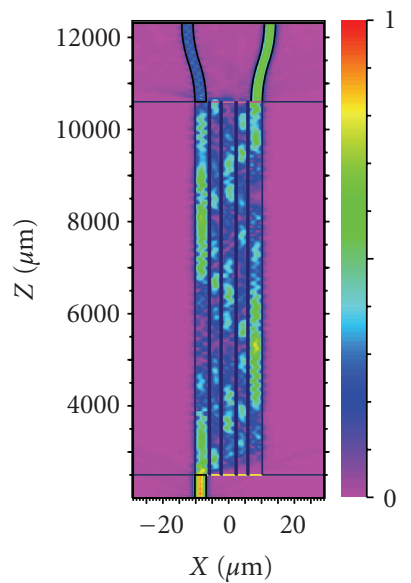

(c)

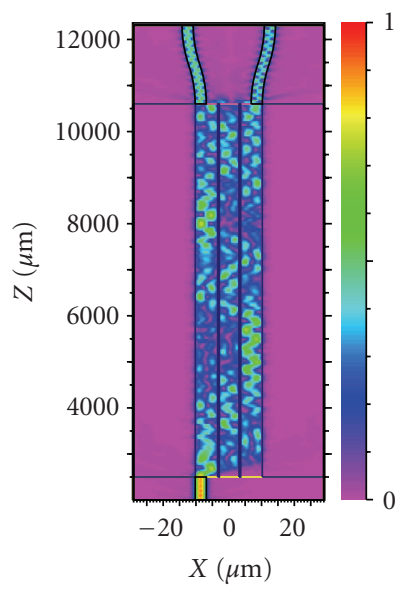

(b)

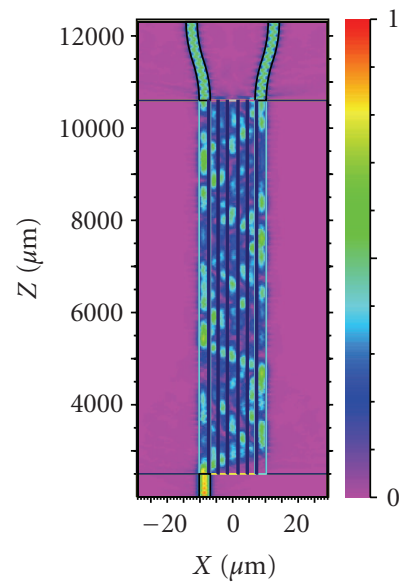

(d)

FIgURE 4: Top view of field distribution in a MMI device with even number of slots: (a)-(d), zero slot to six-slots. 


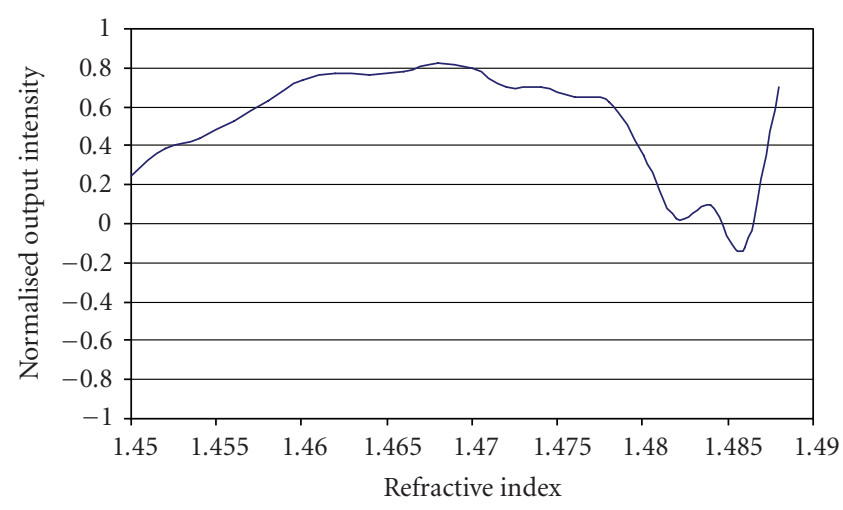

(a)

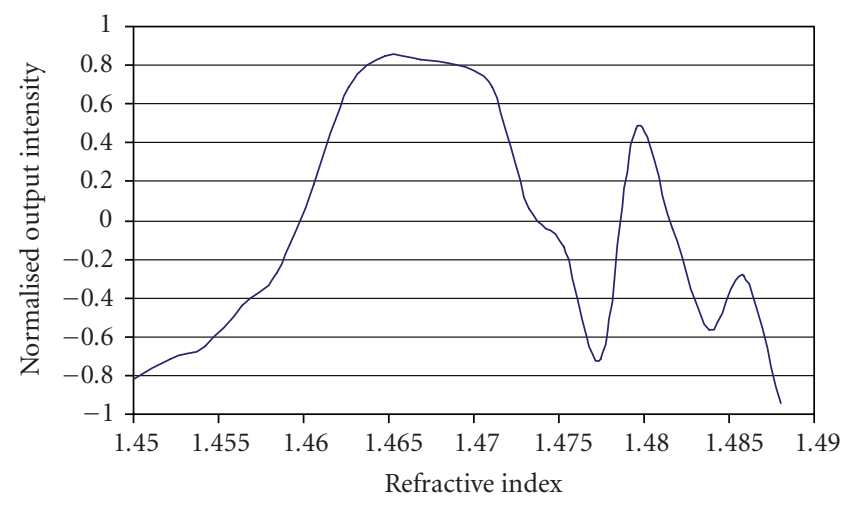

(c)

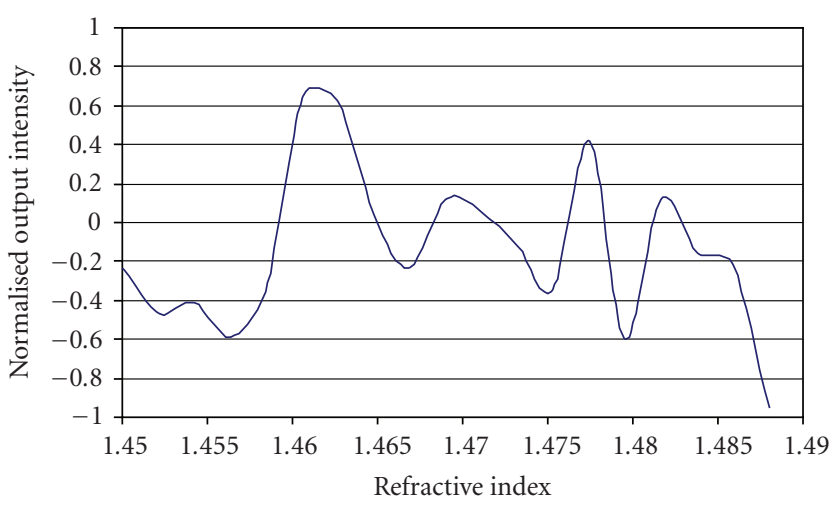

(b)

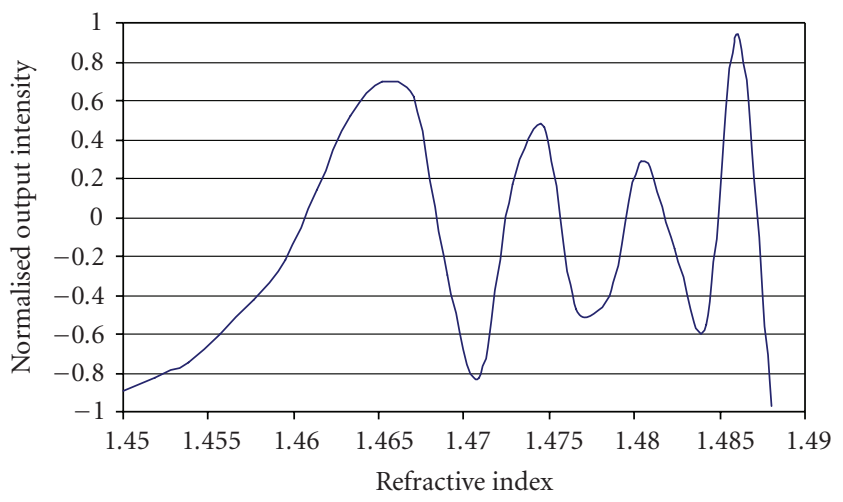

(d)

Figure 5: Normalized differential outputs of (a) zero-, (b) two-, (c) four-, and (d) six-, slot MMI response to different cover liquids refractive index.

occurs whenever the difference in total phase delay between all successive modes is either an odd multiple of $\pi$ resulting in inverted image (cross state) or else an even multiple of $\pi$ resulting in noninverted image (bar state).

Slotted MMIs waveguides have raised a lot of interest due to their potential as MMI devices for separation/combination of wavelength/polarization, powersplitting, and novel types of MMI switches [25] but they have never been used for sensing purposes. This paper investigates the potential sensing application of this class of devices. Figure 3 represents a MMI with six-slots of equal widths which are placed symmetrically relative to the center of device.

In our design, we assumed a glass wafer as the substrate $\left(n_{\text {clad }}=1.445\right)$ and SiON as core material $\left(n_{\text {core }}=1.49\right)$. The fluid film to be analyzed forms the cladding and completely fills the slots. Therefore, we studied the behavior of the device for a typical range of refractive index of many solvents, which ranges between 1.33 for water and 1.5 for protein solution $[26,27]$. The experimental results presented in this paper focuses on materials with index of refraction of around 1.5 and the operating wavelength of $633 \mathrm{~nm}$. Experiments are in progress for the same device dimensions for refractive index of around 1.3 and operating wavelength of $1300 \mathrm{~nm}$. It should be mentioned that wavelengths greater than $1300 \mathrm{~nm}$ are not ideal choices, since most of the liquid-based samples contain water in which they strongly absorb signals at these wavelengths. Slots widths are set to $520 \mathrm{~nm}$ value that can be realized in a reproducible way when using our SiON technology. Since the sensitivity of the sensor is proportional to $L_{\text {sensor }}$, the long sensing structure would be desired. In our design, the length of the device is $8.1 \mathrm{~mm}$. The height of SiON waveguide is optimized to $2.5 \mu \mathrm{m}$.

Every sensor application is characterized by its own specific set of requirements. Because integrated optical sensors are most competitive if high resolution is required, we will focus on this aspect and analyze the resolution prospects of the slotted MMI sensor. Resolution is defined as the smallest change in bulk refractive index that can be detected and measured by the complete sensing system. In slotted MMI sensor, more variation in field distribution has been observed for higher number of slots as the refractive index of sensing material varies.

Figure 4 represents the field propagation in MMIs with even number of slots with the parameters mentioned and $n_{\text {liquid }}$ is set to 1.48 . As depicted in Figure 4, two arbitrary 


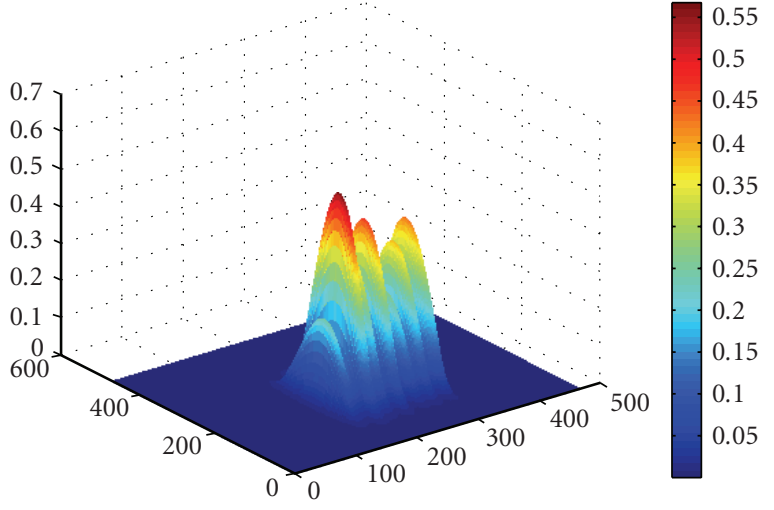

(a)

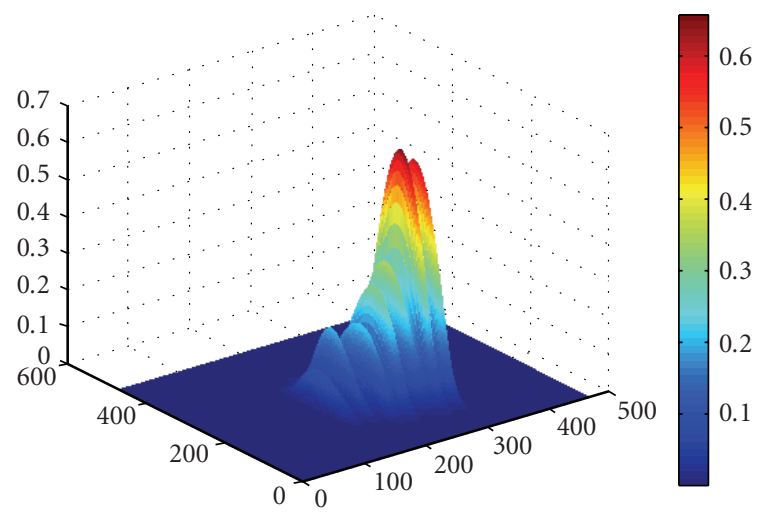

(c)

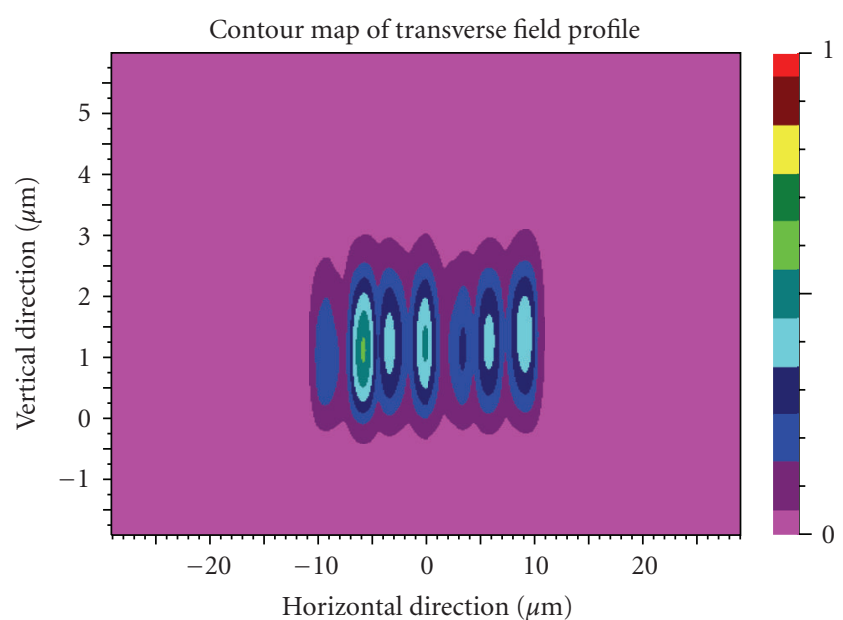

(b)

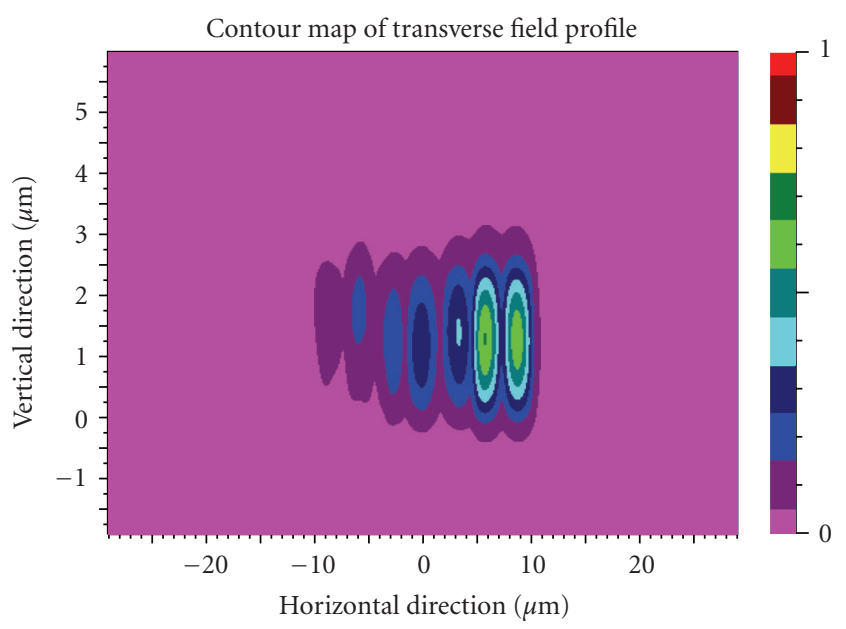

(d)

Figure 6: (a) and (b) 3D power distribution and 2D cross-section image of a Six-slot MMI sensor with cover liquid refractive index of 1.468 at $\lambda=633 \mathrm{~nm}$. (c) and (d) 3D power distribution and 2D cross-section image with cover index of 1.465848 at $\lambda=633 \mathrm{~nm}$. (Horizontal axis and vertical axis are width of the device and output intensity, respectively, with the arbitrary units).

positions at the end face of the slotted MMI sensor have been selected to study the behavior of the field distribution with the change in the refractive index of covering material for different number of slots. The output signal is the relative differential intensity defined as $\Delta P=(P 1-P 2) /(P 1+P 2)$, where $P 1$ is the power in the right output and $P 2$ corresponds to power in the left output. Relative differential intensity was plotted versus refractive indices of sample material and presented in the graph of Figure 5. As the number of slots increases more oscillations will appear in the output signal, corresponding to an increase in responsivity of sensor for a small range of refractive index. The most oscillations have been detected for the highest number of slots. For six-slot MMI we can distinguish three maxima and minima, which oscillate from -1 to 1 in a small range of refractive index where 1 differential output corresponds to all power in right- hand output port of MMI and -1 corresponds to all power in left-hand port.

Rather than limiting ourselves to only two data points in the output side, we could monitor the intensity distribution at every point of the output face. Figure 6 represents the intensity distribution at the endface of a six-slot MMI sensor with the material parameters and dimensions as defined above while the refractive index of top liquids is 1.4682 and 1.465848. Figures 6(a) and 6(c) present a 3D intensity profile at the output end of device if the refractive indexes of sensing materials are 1.4682 and 1.465848 , respectively. Figures 6(b) and 6(d) represent the cross-section of intensity distribution for two different refractive indexes of 1.4682 and 1.465848 , respectively. A significant change in the output profile could be observed only if the refractive index of the covering material was changed by $2 \times 10^{-3}$. 


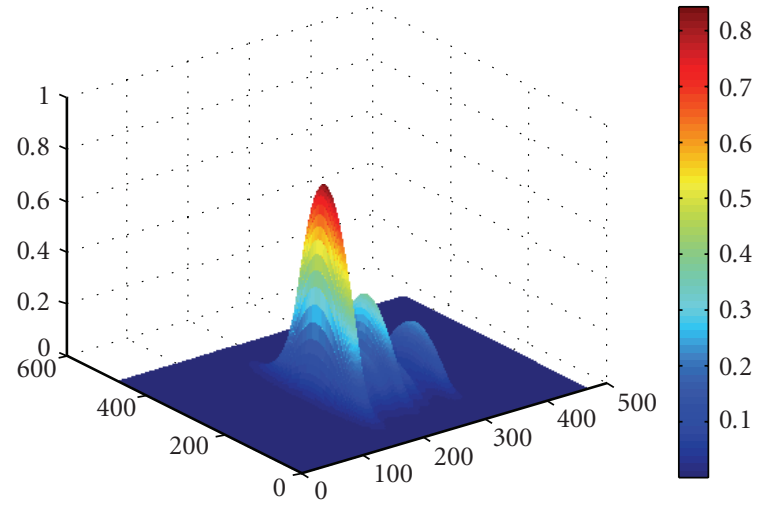

(a)

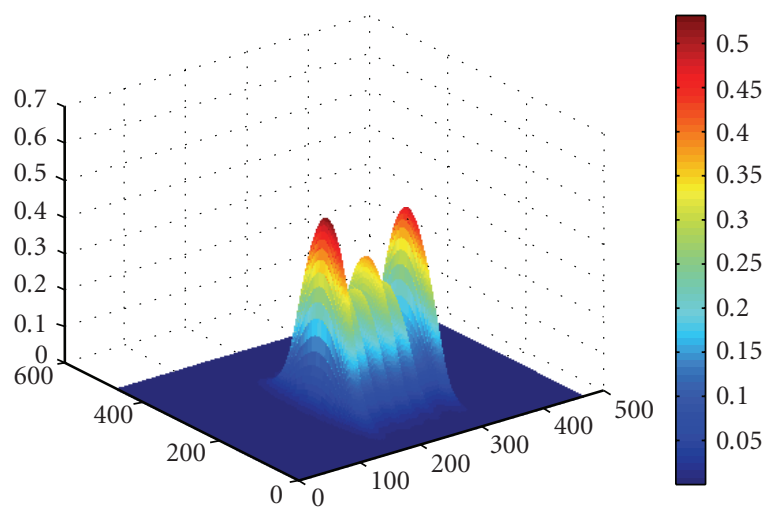

(c)

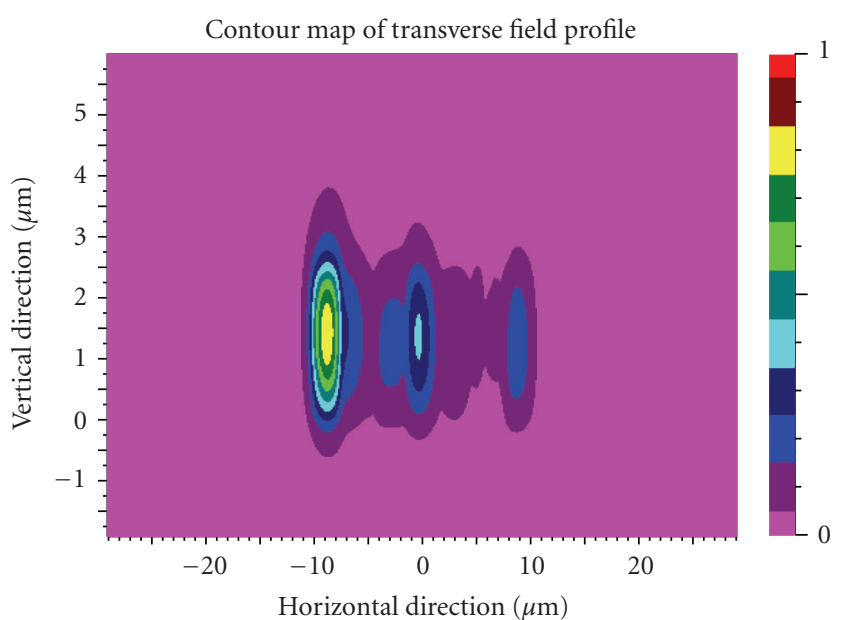

(b)

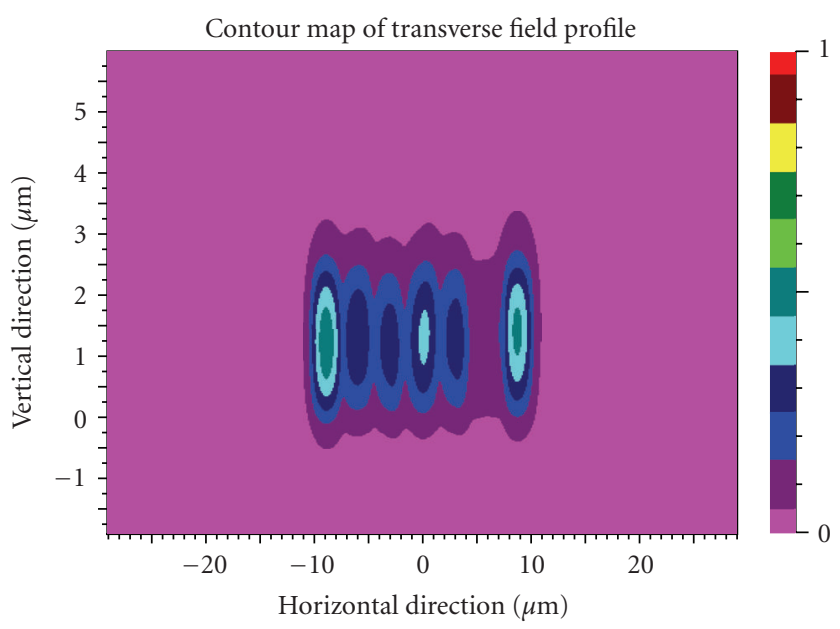

(d)

Figure 7: (a) 3D power distribution and (b) 2D cross-section image of a six-slot MMI sensor with cover liquid refractive index of 1.468 at $\lambda=780 \mathrm{~nm}$. (c) $3 \mathrm{D}$ power distribution and (d) $2 \mathrm{D}$ cross-section with cover index of 1.465848 at $\lambda=780 \mathrm{~nm}$.

As Figure 5 shows, the response of slotted MMI devices is periodic in general which could lead to measurement ambiguity. In order to resolve this potential problem, we have investigated a wavelength interrogation method in which more than one wavelength at any time should be used so that the combination of sensor response at these wavelengths would lead to a unique value of refractive index.

In order to analyze this solution we simulated the behavior of the six-slotted MMI device used to obtain the results of Figure 6 at two new wavelengths. Therefore, we maintained the same material properties, dimensions, and covering liquid indices; however, the operating wavelengths are of $780 \mathrm{~nm}$ and $800 \mathrm{~nm}$.

The results are illustrated in Figures 7 and 8.Comparison of the results of Figures 8 and 9 illustrates that for any given refractive index the near field intensity profile of the sensor output depends on the operating wavelength. However, when the intensity profiles at two wavelengths of
$780 \mathrm{~nm}$ and $800 \mathrm{~nm}$ are simultaneously obtained, a unique and unambiguous value for the index of refraction could be determined.

Further investigations showed that this device is capable of detecting very small variation of refractive index. A very simple signal detection scheme could conservatively recognize index variation in the order of $10^{-5}$. Graph in Figure 9 represents the normalized differential output intensity between the first and sixth waveguides (from left to right) as the refractive index of cover liquid changes between 1.465652 and 1.466436 with step size of $9.8 \times 10^{-5}$. The theoretical predictions agree well with the experimental results, as will be demonstrated later.

\section{Fabrication}

Different technologies are being employed for producing IO sensors which depend on the application. The SiON 


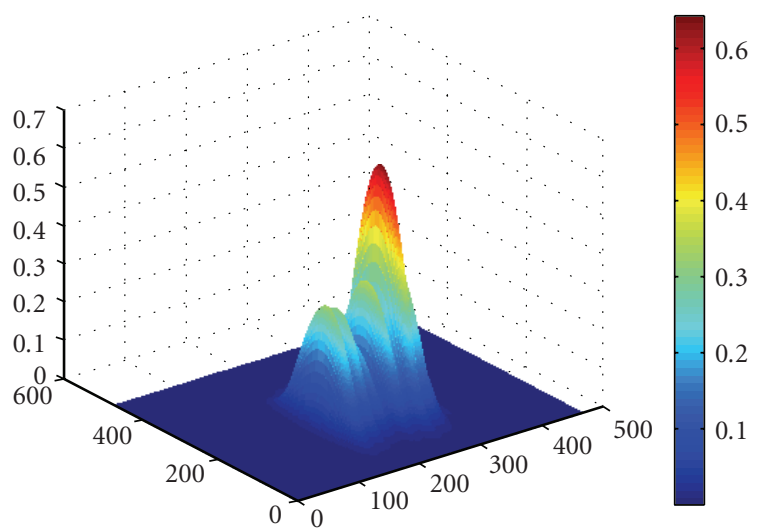

(a)

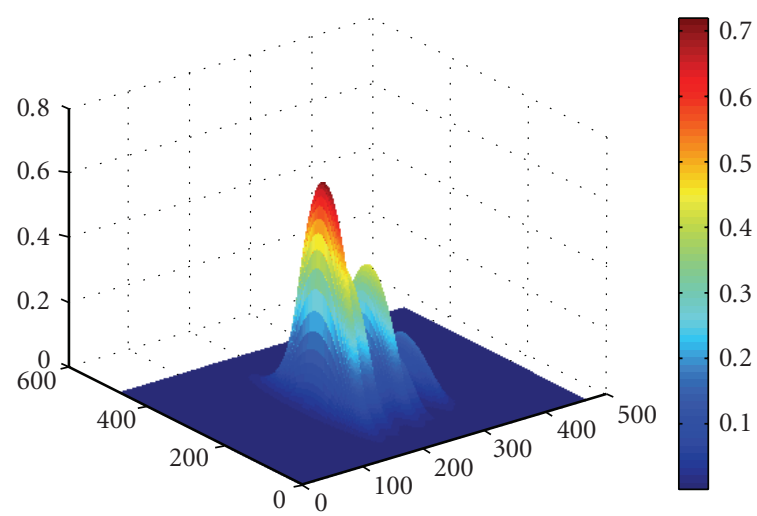

(c)

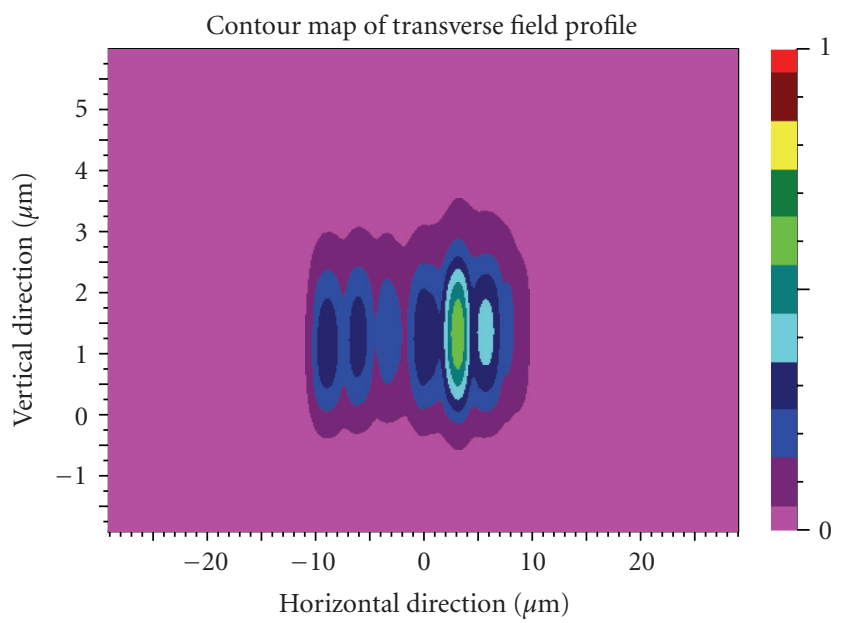

(b)

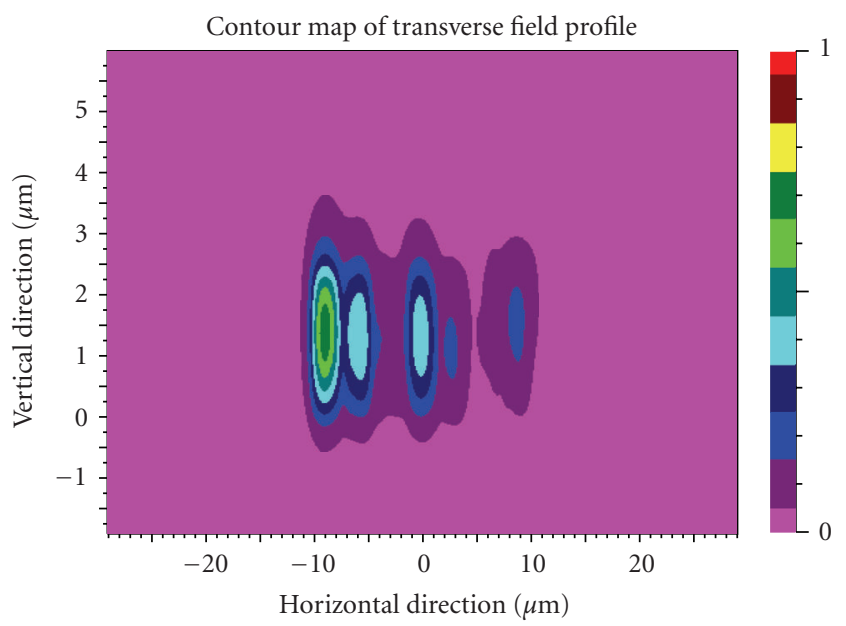

(d)

Figure 8: (a) 3D power distribution and (b) 2D cross-section image of a Six-slot MMI sensor with cover liquid refractive index of 1.468 at $\lambda=800 \mathrm{~nm}$. (c) $3 \mathrm{D}$ power distribution and (d) $2 \mathrm{D}$ cross-section image with cover index of 1.465848 at $\lambda=800 \mathrm{~nm}$.

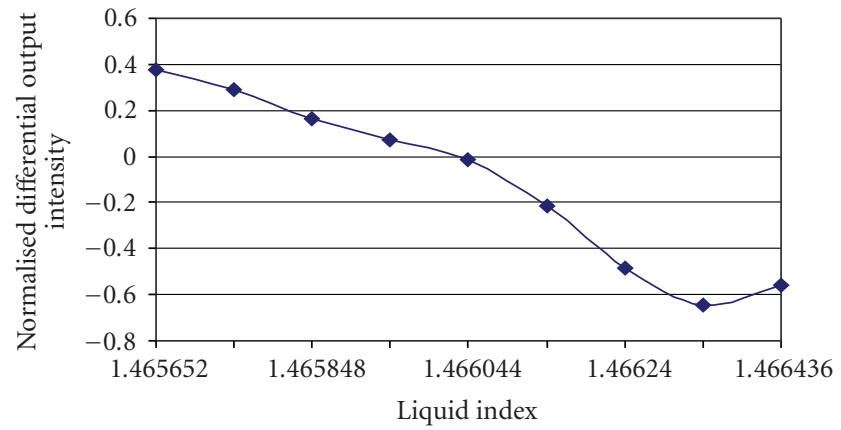

Figure 9: Normalized differential output intensity between waveguides one and six (from left to right) versus refractive index of liquid $(\lambda=633 \mathrm{~nm})$. 


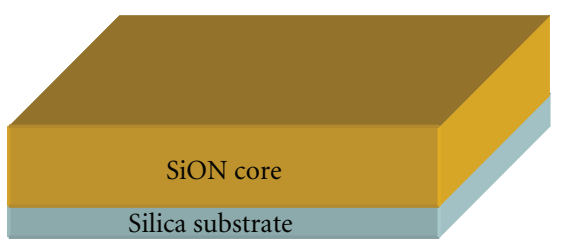

(a) Growth SiON layer by PECVD.

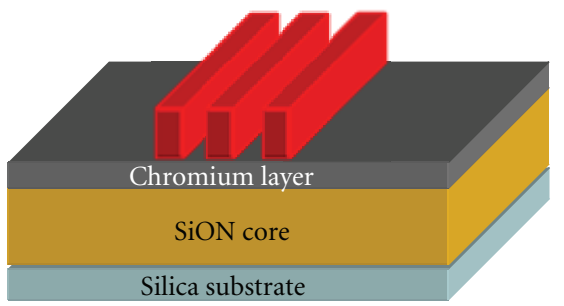

(d) Develop photoresist.

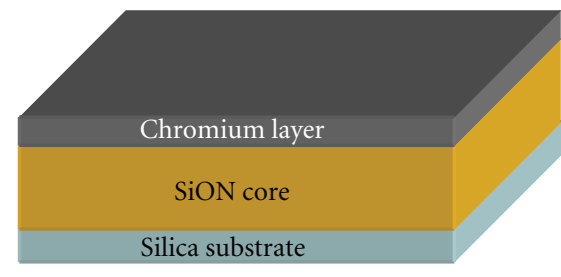

(b) Deposition of chromium layer by PVD.

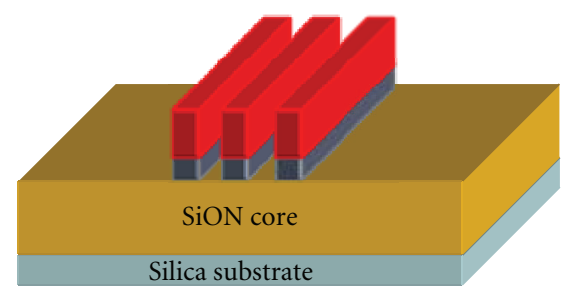

(e) Chromium RIE.

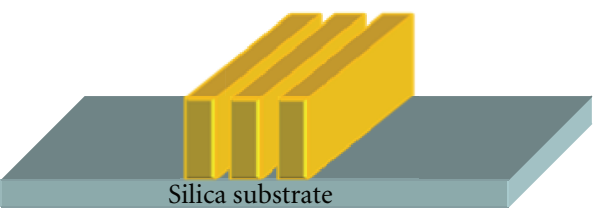

(g) Wet chromium etch.

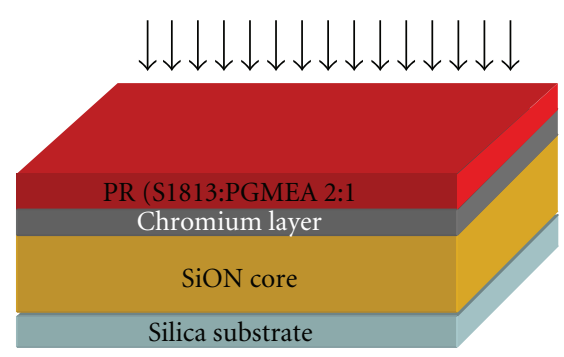

(c) Photolithography.

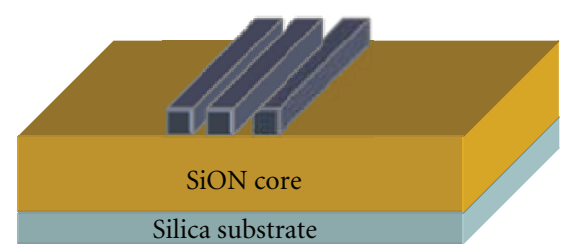

(f) SiON layer RIE.

Figure 10: Fabrication process of slotted MMI sensor.

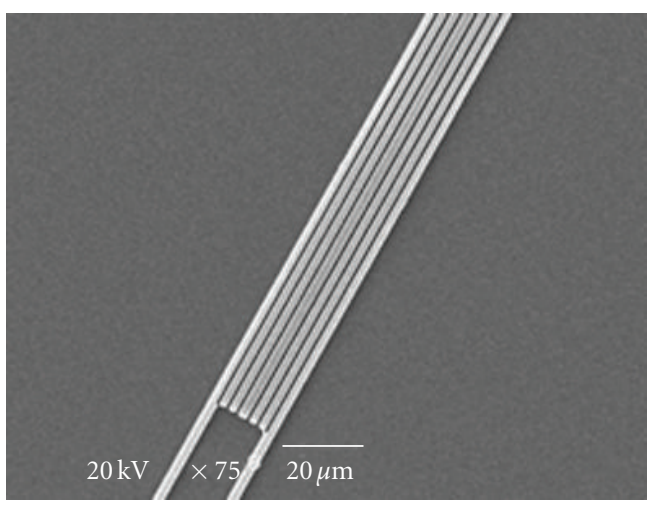

(a)

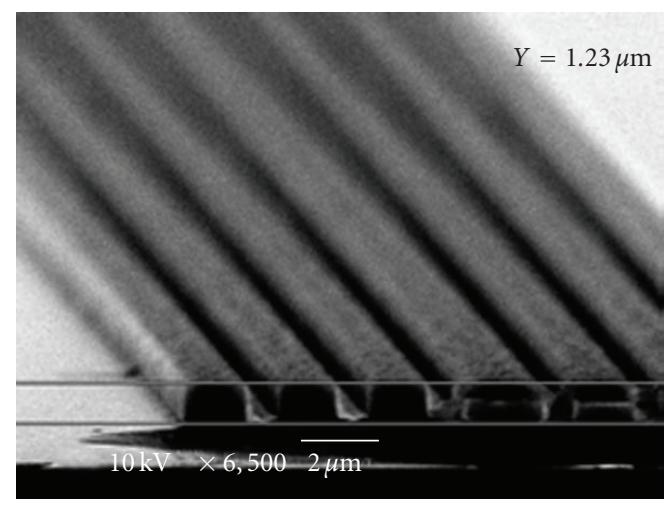

(b)

Figure 11: SEM view of slotted MMI device. (a) Top view: $750 \times$, (b) cross-section $6500 \times$.

technology [28-30], indiffusion of glass [31], and polymer technology [32] are few to name. Between them, the SiON technology is the large range of wavelengths from visible to near infrared. With a precise control of process during reactive ion etching (RIE), SiON provides us with a high quality, straight sidewall which is required to our application. The chemical inertness of SiON makes this material a good candidate to avoid the reactivity with biological agents and processing chemicals. Finally, SiON growth and processing parameters have been well studied so that the desired characteristics required for this proposed sensor could be readily achieved.

Sensor is structured in the multilayer stacks deposited on top of a silica substrate. SiON has been deposited by plasma enhanced chemical vapor deposition (PECVD), and the refractive index has been tailored by mixing different gas concentrations $\left(\mathrm{SiH}_{4}, \mathrm{~N}_{2} \mathrm{O}\right.$, and $\left.\mathrm{NH}_{3}\right)$. Patterns are created by photolithography and have been developed and etched by RIE using $\mathrm{C}_{4} \mathrm{~F}_{8}$ and $\mathrm{O}_{2}$. Figure 10 represents the fabrication process of slotted MMI sensor which will be discussed 

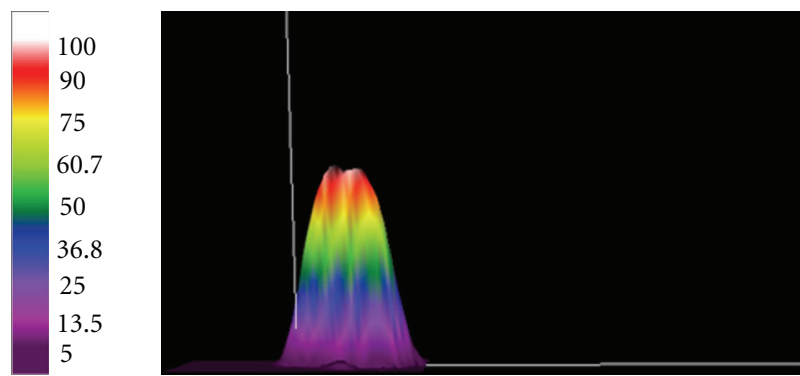

Clip levels (\%)

Figure 12: 3D beam profiler image of 6-slot MMI sensor at wavelength of $633 \mathrm{~nm}$ and liquid refractive index of 1.465652 covering the sensor. (Horizontal axis and vertical axis are MMI width and intensity distribution along the MMI width with arbitrary units).

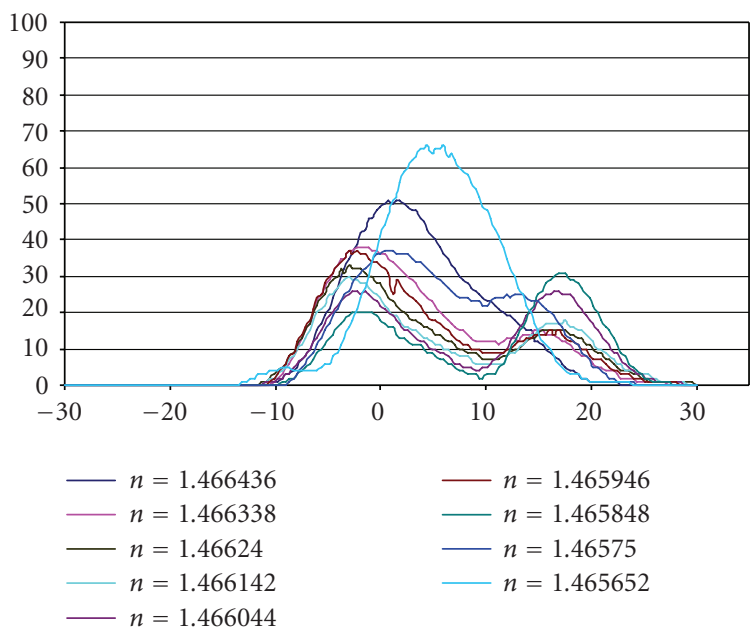

Figure 13: Power distribution along the endface of 6 slot MMI sensor for 9 different refractive indexes of cover medium. . (Horizontal and vertical axis are MMI width and output intensity, respectively, with arbitrary units).

in more details as we proceed. First a $2.5 \mu \mathrm{m}$-thick SiON waveguide layer with refractive index of 1.49 was grown on a quartz wafer using PECVD. A thin layer of chromium deposited on SiON using PVD-Lesker 75 tools to a thickness of $120 \mathrm{~nm}$. To define the slotted MMI, a positive photoresist S1813 diluted in PGMEA with the ratio 2: 1 was spun on the chromium layer. Pattern has been transferred by a stepper tool, with a projection aligner with a reduction factor of 5 . After exposure and development of the resist, the pattern was transferred into the $120 \mathrm{~nm}$-thick chromium layer by liftoff. The resist layer acted as an etch mask for the underlying $\mathrm{Cr}$ layer by reactive ion etching (RIE) in $\mathrm{O}_{2}-\mathrm{Cl}_{2}$ plasma. The chief advantage of dry $\mathrm{Cr}$. etch is that it etches anisotropically, which significantly reduces the chrome etch undercut. As a consequence, the critical dimension (CD) bias is greatly reduced and resolution improves [33]. Finally the Cr layer was used as a mask in a second RIE step to transfer the slotted MMI structure into the SiON waveguide layer. C4F8/O2, $80 / 5$ sccm-based plasmas are used for selective etching of

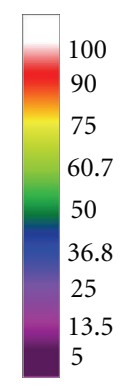

Clip levels (\%)

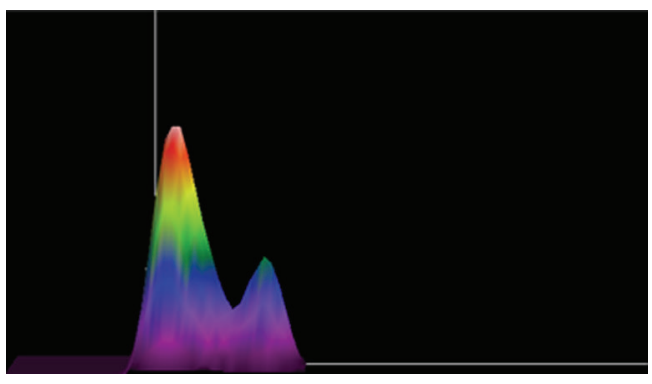

(a)
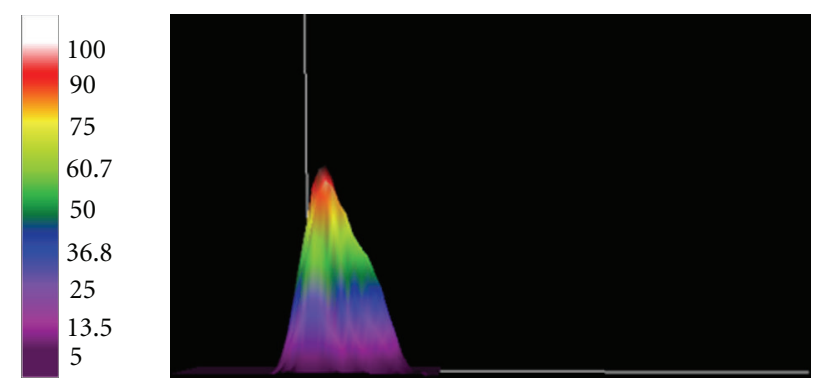

Clip levels (\%)

(b)

Figure 14: Three-dimensional far-field intensity distribution of output of six-slot MMI for refractive index of liquid (a) 1.466240 and (b) 1.466436 (Wavelength of $633 \mathrm{~nm}$ ). (Horizontal axis and vertical axis are MMI width and intensity distribution along the MMI width, respectively, with arbitrary units).

high aspect ratio trenches in $\mathrm{SiON}$. Additives such as oxygen are often used to optimize the process $[34,35]$. In the last step fused silica wafers were cut in $1 \mathrm{in}^{2}$ chips with a highspeed saw, followed by a polishing step. Some Scanning Electron Microscope (SEM) pictures of the devices are shown in Figure 11. The cross-section of device shows an almost perfect rectangular trenches and straight, smooth side walls.

\section{Experiments}

Each chip containing 10 devices was mounted on a translation stage. Light from a $\mathrm{He}-\mathrm{Ne}$ at $633 \mathrm{~nm}$ was launched into a single mode fiber and coupled to the input waveguide of the MMI sensor. The light output from sensor was imaged on a BeamPro (Photon Inc.) camera. Over the depth of focus of the imaging lens the output signal is integrated; therefore, the intensity profile obtained with this method should be considered to be the far-field distribution of the device, while the simulations of Figures 6, 7, and 8 represent the near-field intensity profile.

A liquid with refractive index of 1.4682 at $633 \mathrm{~nm}$ wavelength and temperature coefficient of $0.000392 /{ }^{\circ} \mathrm{C}$ (Gargille certified) were used as sensing material. The cover refractive index was changed in very small steps between 1.4632 and 1.4682 by an infrared thermal emitter source element. BeamProfiler software (version 2.84) was used to monitor the 
far-field intensity distribution as the refractive index changes and is compared with values published in literatures [220]. Figure 12 shows a 3D far-field intensity profile of the output end of six-slot MMI sensor with the dimensions and device parameters mentioned in fabrication section with the cover liquid's refractive index of 1.465652 at the wavelength $633 \mathrm{~nm}$.

The change in refractive index has been carried out by heating the covering medium using infrared thermal emitter source, while real-time intensity change of the output endface is being monitored and stored in very small steps by camera and software. Figure 13 plots power distribution along the MMI width at the wavelength of $633 \mathrm{~nm}$ for nine different refractive indices with $\delta n_{\text {cover }}=9.8 \times 10^{-5}$. For the sake of clarity, not all $n$ measured are shown in this Figure. Graphs show a noticeable change in distribution of power, as the refractive index of cover medium changes by the order of $10^{-5}$.

Figure 14 shows a 3D image of intensity distribution for two different cover indices in the far-field. As depicted, the intensity distribution pattern at the output shifts as the refractive index increases from (a) 1.466240 to (b) 1.466436.

Results of Figure 13 verify a resolution of the order of $10^{-5}$ for change in the refractive index, as estimated in the theoretical section. This resolution implies a control of the temperature of the sensor and liquid system within $0.2^{\circ} \mathrm{C}$, assuming typical thermo-optical coefficients of liquids of interest in biochemical and chemical monitoring. This could be overcome by developing a calibration process and implementing a dual wavelengths interrogation method. Although the results presented in this paper are for the refractive indices of around 1.46, this device could be easily used for much lower refractive index range, around 1.33, to detect water-soluable chemical. In such a case all the device parameters and dimensions should remain the same, but the operation wavelength would be at $1300 \mathrm{~nm}$. This wavelength is below the absorption spectrum of water.

At this point no assessment of the repeatability of the fabrication process has been done, but all devices on a single chip were functional. Further study should be conducted to compare their performance characteristics. Another aspect of this project that requires further investigation is the development of a practical calibration method. As Figures 68 illustrate, the dynamic range of slotted MMI sensors can be enhanced if two or more wavelengths are incorporated to overcome the ambiguity problem. Another solution that is being considered is to develop an image processing tool to identify the power distribution associated with a unique index of refraction.

\section{Conclusion}

A novel configuration of integrated optical sensors based on slotted MMI structures has been proposed and demonstrated. The results confirmed that higher number of slots in the multimode area increases the sensitivity of device to the change in bulk refractive index of sensing species. Material of choice was $\mathrm{SiON}$ which is a robust material in terms of interaction with detectable chemicals comparing to sol-gels, and the technology for depositing $\mathrm{SiON}$ has been already developed. A maximum resolution of $9.8 \times 10^{-5}$ RIU at a specific operating regime is expected.

\section{References}

[1] P. V. Lambeck, J. van Lith, and H. J. W. M. Hoekstra, "Three novel integrated optical sensing structures for the chemical domain," Sensors and Actuators B, vol. 113, no. 2, pp. 718-729, 2006.

[2] R. G. Heideman, R. P. H. Kooyman, and J. Greve, "Performance of a highly sensitive optical waveguide Mach-Zehnder interferometer immunosensor," Sensors and Actuators B, vol. 10, no. 3, pp. 209-217, 1993.

[3] R. G. Heideman and P. V. Lambeck, "Remote opto-chemical sensing with extreme sensitivity: design, fabrication and performance of a pigtailed integrated optical phase-modulated Mach-Zehnder interferometer system," Sensors and Actuators $B$, vol. 61, no. 1, pp. 100-127, 1999.

[4] B. Drapp, J. Piehler, A. Brecht, et al., "Integrated optical MachZehnder interferometers as simazine immunoprobes," Sensors and Actuators B, vol. 39, no. 1-3, pp. 277-282, 1997.

[5] G. L. Duveneck, M. Pawlak, D. Neuschäfer, et al., "Novel bioaffinity sensors for trace analysis based on luminescence excitation by planar waveguides," Sensors and Actuators B, vol. 38, no. 1-33, pp. 88-95, 1997.

[6] P. Hua, B. J. Luff, G. R. Quigley, J. S. Wilkinson, and K. Kawaguchi, "Integrated optical dual Mach-Zehnder interferometer sensor," Sensors and Actuators B, vol. 87, no. 2, pp. 250257, 2002.

[7] A. Brandenburg, "Differential refractometry by an integratedoptical Young interferometer," Sensors and Actuators B, vol. 39, no. 1-3, pp. 266-271, 1997.

[8] G. H. Cross and Y. Ren, "Youngs fringes from vertically integrated slab waveguides: applications to humidity sensing," Journal of Applied Physics, vol. 86, no. 11, pp. 6483-6488, 1999.

[9] A. Ymeti, J. S. Kanger, J. Greve, P. V. Lambeck, R. Wijn, and R. G. Heideman, "Realization of a multichannel integrated Young interferometer chemical sensor," Applied Optics, vol. 42, no. 28, pp. 5649-5660, 2003.

[10] W. Faubel, B. S. Seidel, and H. J. Ache, "Trace analysis of water pollutants by photothermal phase shift spectroscopy with an integrated optical micro-interferometer," Optical Engineering, vol. 35, no. 12, pp. 3555-3561, 1996.

[11] X. Fan, I. M. White, H. Zhu, J. D. Suter, and H. Oveys, "Overview of novel integrated optical ring resonator bio/chemical sensors," in Laser Resonators and Beam Control IX, vol. 6452 of Proceedings of SPIE, San Jose, Calif, USA, January 2007.

[12] C. A. Barrios, K. B. Gylfason, B. Sánchez, et al., "Slotwaveguide biochemical sensor," Optics Letters, vol. 32, no. 21, pp. 3080-3082, 2007.

[13] E. Krioukov, D. J. W. Klunder, A. Driessen, C. Otto, and J. Greve, "Refractive index sensing using an integrated optical microcavity," Optics Letters, no. 7, pp. 512-514, 2002.

[14] J. Homola, S. S. Yee, and G. Gauglitz, "Surface plasmon resonance sensors: review," Sensors and Actuators B, vol. 54, no. 1, pp. 3-15, 1999.

[15] G. Nemova, A. V. Kabashin, and R. Kashyap, "Surface plasmon-polariton Mach-Zehnder refractive index sensor," Journal of the Optical Society of America B, vol. 25, no. 10, pp. 1673-1677, 2008. 
[16] X. Wu, J. Zhang, J. Chen, C. Zhao, and Q. Gong, "Refractive index sensor based on surface-plasmon interference," Optics Letters, vol. 34, no. 3, pp. 392-394, 2009.

[17] S. Grego, J. R. McDaniel, and B. R. Stoner, "Wavelength interrogation of grating-based optical biosensors in the input coupler configuration," Sensors and Actuators B, vol. 131, no. 2, pp. 347-355, 2008.

[18] K. Cottier, M. Wiki, G. Voirin, H. Gao, and R. E. Kunz, "Label-free highly sensitive detection of (small) molecules by wavelength interrogation of integrated optical chips," Sensors and Actuators B, vol. 91, no. 1-3, pp. 241-251, 2003.

[19] J. Piehler, A. Brandenburg, A. Brecht, E. Wagner, and G. Gauglitz, "Characterization of grating couplers for affinitybased pesticide sensing," Applied Optics, vol. 36, no. 25, pp. 6554-6562, 1997.

[20] http://www.microvacuum.com.

[21] A. Irace and G. Breglio, "All-silicon optical temperature sensor based on Multi-Mode Interference," Optics Express, vol. 11, no. 22, pp. 2807-2812, 2003.

[22] K. R. Kribich, R. Copperwhite, H. Barry, et al., "Novel chemical sensor/biosensor platform based on optical multimode interference (MMI) couplers," Sensors and Actuators B, vol. 107, no. 1, pp. 188-192, 2005.

[23] D. A. May-Arrioja, P. L. Wa, J. J. Sánchez-Móndragon, R. J. Selvas-Aguilar, and I. Torres-Gomez, "A reconfigurable multimode interference splitter for sensing applications," Measurement Science \& Technology, vol. 18, no. 10, pp. 32413246, 2007.

[24] L. B. Soldano and E. C. M. Pennings, "Optical multi-mode interference devices based on self-imaging: principles and applications," Journal of Lightwave Technology, vol. 13, no. 4, pp. 615-627, 1995.

[25] D. M. Mackie and A. W. Lee, "Slotted multimode-interference devices," Applied Optics, vol. 43, no. 36, pp. 6609-6619, 2004.

[26] M. Noto, M. Khoshsima, D. Keng, I. Teraoka, V. Kolchenko, and S. Arnold, "Molecular weight dependence of a whispering gallery mode biosensor," Applied Physics Letters, vol. 87, no. 22, Article ID 223901, 3 pages, 2005.

[27] H. El-Kashef, "Necessary requirements imposed on polar dielectric laser dye solvents," Physica B, vol. 279, no. 4, pp. 295$301,2000$.

[28] K. Wörhoff, P. V. Lambeck, and A. Driessen, "Design, tolerance analysis, and fabrication of silicon oxynitride based planar optical waveguides for communication devices," Journal of Lightwave Technology, vol. 17, no. 8, pp. 1401-1407, 1999.

[29] K. Wörhoff, P. V. Lambeck, H. Albers, O. F. Noordman, N. F. Hulst, and Th. J. Popma, "Optimization of LPCVD silicon oxynitride growth to large refractive index homogeneity and layer thickness uniformity," in Micro-optical Technologies for Measurement, Sensors and Microsystems II and Optical Fiber Sensor Technologies and Applications, vol. 3099 of Proceedings of SPIE, pp. 257-268, Munich, Germany, June 1997.

[30] L. Wosinski, Technology for photonic components in silica/silicon material structure, Ph.D. thesis, Royal Institute of Technology (KTH), Stockholm, Sweden, 2003.

[31] S. I. Najafi, Introduction to Glass Integrated Optics, Artech House, Boston, Mass, USA, 1992.

[32] L. Eldada, "Optical communication components," Review of Scientific Instruments, vol. 75, no. 3, pp. 575-593, 2004.

[33] P. Buck and B. Grenon, "A comparison of wet and dry chrome etching with the CORE-2564," in Photomask Technology and Management, vol. 2087 of Proceedings of the SPIE, 1993.
[34] A. Sankaran and M. J. Kushner, "Etching of porous and solid $\mathrm{SiO}_{2}$ in $\mathrm{Ar} / c-\mathrm{C}_{4} \mathrm{~F}_{8}, \mathrm{O}_{2} / c-\mathrm{C}_{4} \mathrm{~F}_{8}$ and $\mathrm{Ar} / \mathrm{O}_{2} / c-\mathrm{C}_{4} \mathrm{~F}_{8}$ plasmas," Journal of Applied Physics, vol. 97, no. 2, Article ID 023307, 10 pages, 2005.

[35] A. Sankaran and M. J. Kushner, "Integrated feature scale modeling of plasma processing of porous and solid $\mathrm{SiO}_{2}$. I. Fluorocarbon etching," Journal of Vacuum Science and Technology A, vol. 22, no. 4, pp. 1242-1259, 2004. 

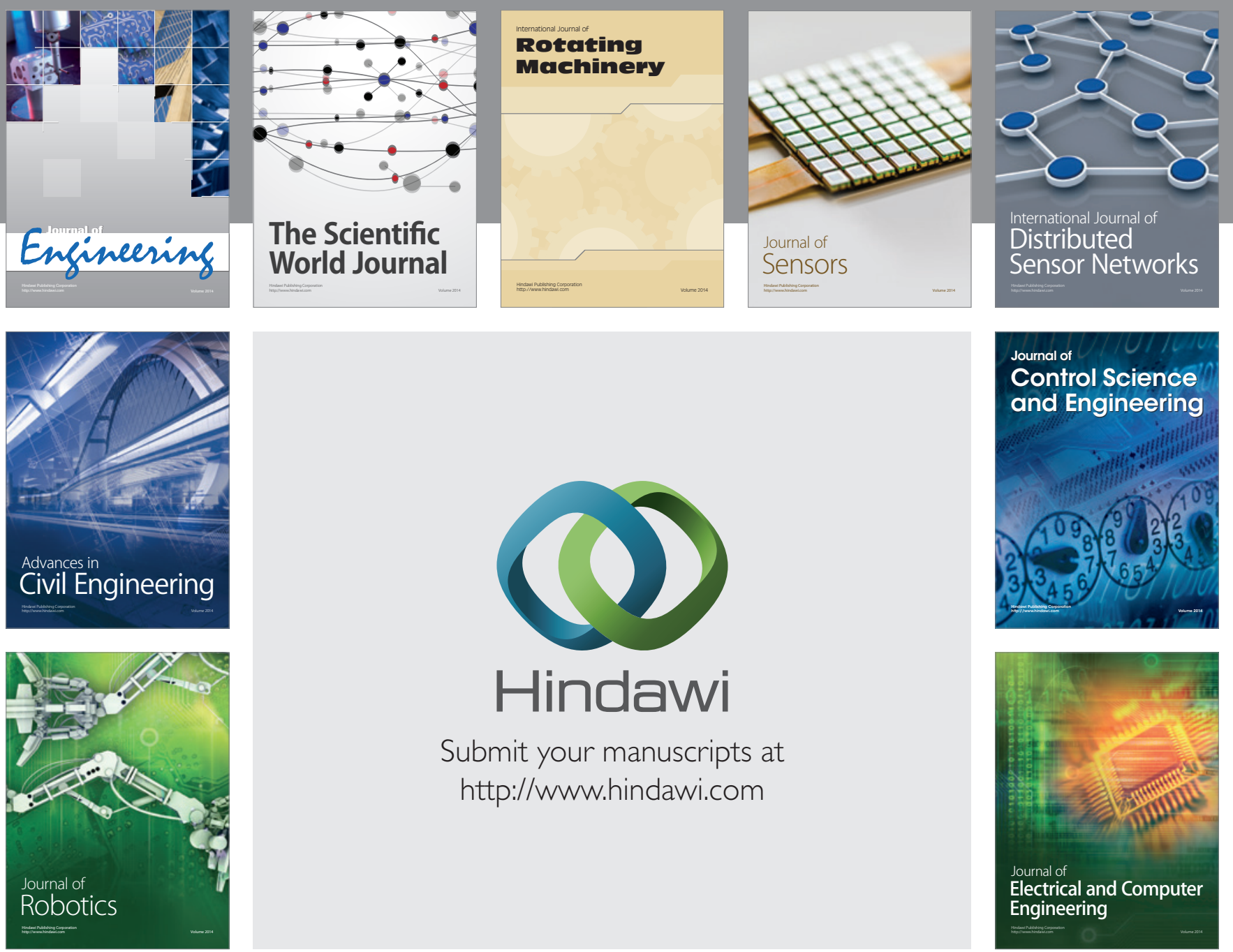

Submit your manuscripts at

http://www.hindawi.com
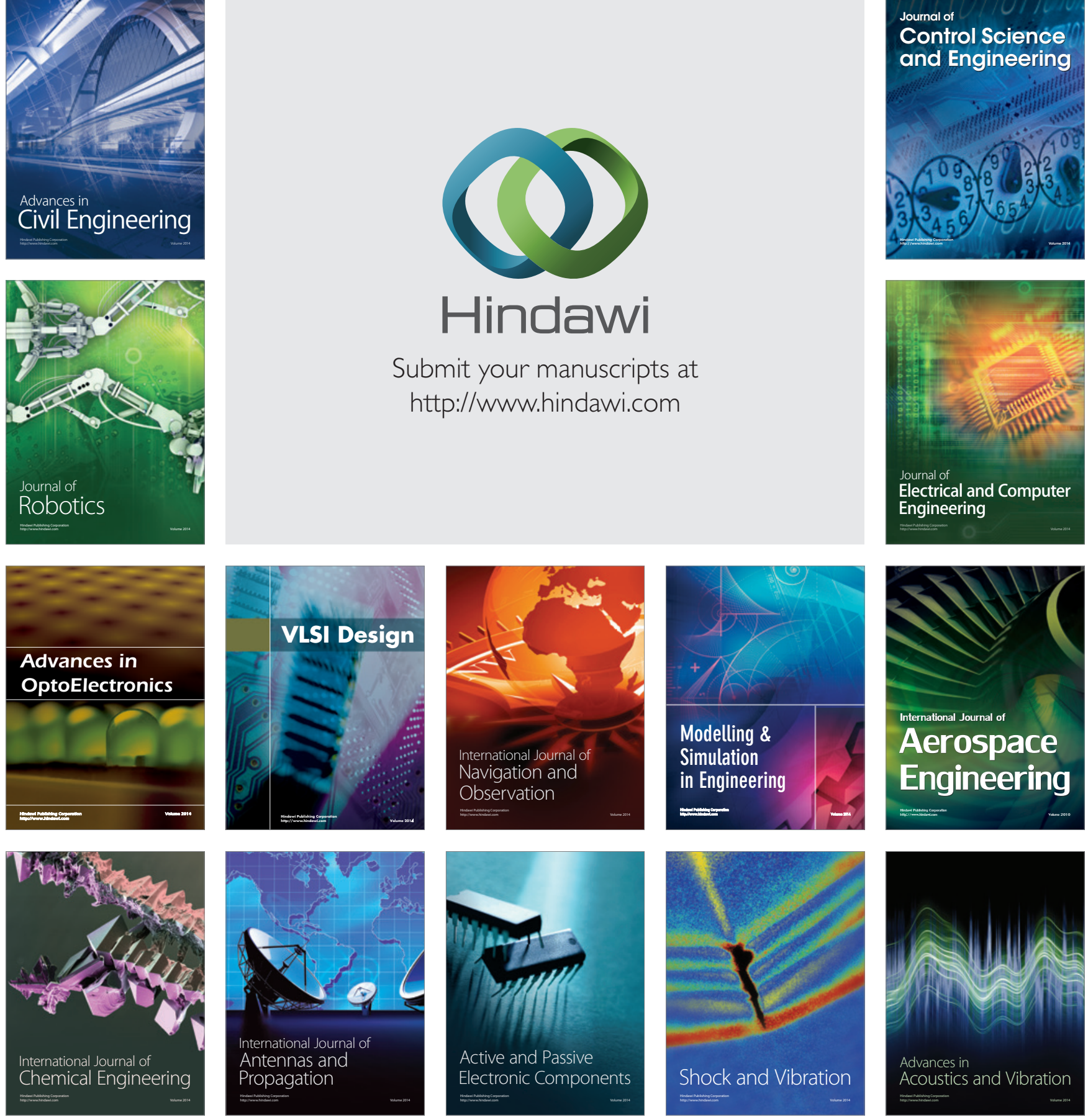